\title{
Left Ventricular and adjacent Right ventricular Pseudoaneurysms post acute myocardial infarction communicating via a Ventricular Septal Defect
}

\author{
Kevin Kang ${ }^{1}$, John Wilson ${ }^{2}$, Jeffrey Friedel ${ }^{2}$, and Angel Flores ${ }^{3}$ \\ ${ }^{1}$ Dartmouth College Geisel School of Medicine \\ ${ }^{2}$ Meadville Medical Center \\ ${ }^{3}$ Butler Memorial Hospital
}

December 16, 2020

\begin{abstract}
The rare but deadly post myocardial infarction (MI) mechanical complications are categorized as ventricular free wall rupture, ventricular septal rupture (VSD) and papillary muscle rupture in decreasing order of incidence. The incidence of the mechanical complications has been mitigated by early revascularization in recent years but mortality remains high. The cardiac rupture if contained by clot, scar and pericardium leads to a LV pseudoaneurysm that delays or diminishes the fatal outcome. Mechanical complications and pseudoaneurysm are recognized by echocardiography. We report a previously unreported occurrence of a pseudoaneurysm involving the adjacent walls of both the ventricles, LV basal and inferoseptal walls and the adjacent right ventricular inferior wall (RV). The LV and RV communicated via a hole in the pseudoaneurysmal wall. The echocardiographic images initially showed the LV pseudoaneurysm bulging into the RV rather than into the pericardium. The color Doppler showed shunting through the LV pseudoaneurysm into the RV creating an unusual VSD. The CT angiograms corroborated the echo findings. The LV pseudoaneurysm had a tear in it and this led to bleeding not into pericardium but into the adjacent RV pseudoaneurysm, hence creating a very unusual VSD. Subsequently, our patient went for surgical repair of pseudoaneurysm and the surgical findings confirmed the imaging findings that there was a massive LV pseudoaneurysm from the inferior and inferoseptal walls, the adjacent RV wall was involved with the pseudoaneurysm and a communication between LV pseudoaneuysm sac and the RV was seen. Such pathology has not been described in the past.
\end{abstract}

\section{TITLE PAGE}

TITLE: Left Ventricular and adjacent Right ventricular Pseudoaneurysms post acute myocardial infarction communicating via a Ventricular Septal Defect

AUTHORS:

1. Kevin Kang BA, MD candidate (corresponding author)

Dartmouth Geisel School Of Medicine, Hanover, NH 03755.

Email: kevinsinghkang@gmail.com

2. John Wilson MD

Meadville Medical Center, Meadville, PA USA.

Email: JWilson1@mmchs.org

3. Jeffrey Friedel MD 
Meadville Medical Center, Meadville, PA USA.

Email: Jfriedel@mmchs.org

4. Angel Flores MD

Butler Hospital, Butler PA USA.

Email: arfa@aol.com

Institution where the work was performed: Meadville Medical Center

Short running head title: LV and RV pseudoaneurysms creating a VSD

Key words: pseudoaneurysm; rupture; septum

Financial Support: None

Type of article: Case Report

Corresponding author's Fax number, telephone number and email address respectively: K. Kang 814-4508160 and kevinsinghkang@gmail.com

Home address: 4368 Winners Circle, Erie PA 16506

Title: Left Ventricle and adjacent Right ventricle Pseudoaneurysms post myocardial infarction communicating via a Ventricular Septal Defect

Abstract

The rare but deadly post myocardial infarction (MI) mechanical complications are categorized as ventricular free wall rupture, ventricular septal rupture (VSD) and papillary muscle rupture in decreasing order of incidence. The incidence of the mechanical complications has been mitigated by early revascularization in recent years but mortality remains high. The cardiac rupture if contained by clot, scar and pericardium leads to a LV pseudoaneurysm that delays or diminishes the fatal outcome. Mechanical complications and pseudoaneurysm are recognized by echocardiography. We report a previously unreported occurrence of a pseudoaneurysm involving the adjacent walls of both the ventricles, LV basal and inferoseptal walls and the adjacent right ventricular inferior wall (RV). The LV and RV communicated via a hole in the pseudoaneurysmal wall. The echocardiographic images initially showed the LV pseudoaneurysm bulging into the RV rather than into the pericardium. The color Doppler showed shunting through the LV pseudoaneurysm into the RV creating an unusual VSD. The CT angiograms corroborated the echo findings. The LV pseudoaneurysm had a tear in it and this led to bleeding not into pericardium but into the adjacent RV pseudoaneurysm, hence creating a very unusual VSD. Subsequently, our patient went for surgical repair of pseudoaneurysm and the surgical findings confirmed the imaging findings that there was a massive LV pseudoaneurysm from the inferior and inferoseptal walls, the adjacent RV wall was involved with the pseudoaneurysm and a communication between LV pseudoaneuysm sac and the RV was seen. Such pathology has not been described in the past.

\section{Manuscript}

Introduction:

Acute myocardial infarction can be associated rarely with deadly mechanical complications resulting from myocardial rupture $(1,2)$. The types of mechanical complications are categorized as ventricular free wall rupture (FWR), ventricular septal rupture (VSD) and papillary muscle rupture (PMR) in decreasing order of incidence $(2,3)$. The FWR has been reported in up to $10 \%$ (dropping to $2 \%$ currently) of Mis in prereperfusion era while VSD and PMR had a lower incidence of $1-2 \%$ and $0.5-5 \%$, respectively (3). The risk of these complications has been reduced by early revascularization, but the consequences stay devastating with very high mortality. Left ventricular free wall rupture has very high mortality post myocardial infarction (MI) but if the left ventricle (LV) is not communicating freely with pericardium, the consequences are mitigated 
and mortality delayed or reduced. This can happen due to formation of a pseudoaneurysm (PSA) at the site of rupture. The tear in the myocardial wall is contained by the pseudoaneurysm wall, which is formed by the adherent clot, scar tissue and adjacent visceral pericardium that balloons out into the parietal pericardium (4). This process stops the left ventricular bleeding because the cardiac rupture is contained by adherent pericardium or scar tissue, which are forming the thin pseudoaneurysm wall (4). Thus, unlike a true LV aneurysm, a LV pseudoaneurysm contains no endocardium or myocardium (4). Wall stress, which is related to LV pressure, LV radius, and importantly LV wall thickness along with the loss of myocardial integrity (from myocardial infarction) are the likely determinants of the subsequent cardiac rupture and free catastrophic bleeding into pericardial cavity (5). As noted, most commonly, the initial free wall intra-pericardial rupture results in cardiac tamponade and sudden death but less frequently, cardiac rupture is contained and LV pseudoaneurysm formation occurs $(6,7)$. However, in a patient presenting with normal blood pressure and contained free wall rupture, the diagnosis of LV pseudoaneurysm is difficult as it is uncommon but also costly to miss because of the near certain mortality of the rupture of the pseudoaneurysm wall $(8,9)$. Post-infarct ventricular septal defect (PIVSD) can be another devastating complication of acute myocardial infarction (MI). In the pre-reperfusion era, the VSD prevalence was $1 \%$ to $3 \%$, dropping to $0.2 \%$ with reperfusion (6). Both ventricular septal defect (VSD) and pseudoaneurysms can be recognized by echocardiography (10). We present a case of a pseudoaneurysm involving both the basal inferoseptal wall of the left ventricle and the adjacent right ventricle (RV) along with an unusual VSD. The left ventricle communicated with the right ventricle (RV) through the pseudoaneurysm sac.

Case Presentation:

66-year-old male was a cardiology outpatient on June 19, 2018 for chest pressure that he experienced on May 26, 2018. An electrocardiogram (ECG) from June 11, 2018 showed evidence of age indeterminate inferior myocardial infarction. Stress perfusion imaging was done on June 25, 2018 and was low risk. He walked over 6 minutes on Bruce protocol and the test showed inferior-septal infarct and no ischemia. Two weeks later, his symptoms worsened, and he came to cardiology office on July 13, 2018. A new holosystolic murmur was heard. An emergent echocardiogram showed a ventricular septal defect (VSD) and pseudoaneurysm involving the basal inferior wall. An emergent coronary angiogram was done in our referring hospital that showed that the right coronary artery was occluded, and this was left occluded. An intra-aortic balloon pump was placed. Right heart catheterization or left ventricular angiogram was not done. The patient was then transferred to our tertiary care hospital that has open-heart surgery capability.

A repeat echocardiogram at our institution was done upon arrival. 4-chamber showed a large pseudoaneurysm that involved the inferoseptal wall (Figure 1 and Video 1). Several echocardiographic views revealed that the pseudoaneurysm bulged into the RV rather than the pericardium only (Figure 2 and Video 2). The pseudoaneurysm, despite taking several views, was never seen without also visualizing the adjacent right ventricle leading to the eventual surgical findings showing that the even the right ventricle adjacent to the inferior septum was pseudoaneurysmal. A VSD had been formed between the LV pseudoaneurysm sac and the adjacent right ventricular pseudoaneurysm sac. The VSD was noted by color Doppler showing that the flow into the pseudoaneurysm sac could be followed through a tear in the pseudoaneurysm wall into the right ventricle with associated moderate tricuspid regurgitation seen as well (Figures 3 and Video 3). The impingement from LV pseudoaneurysm and also the VSD jet may have contributed to the tricuspid regurgitation jet (Figure 4 and Video 4). Again, this was confirmed by surgical findings even though the saline bubble injection failed to show communication between left ventricle and right ventricle. Indeed, the bubbles were entering the pseudoaneurysm chamber through the narrow-necked tear in the inferoseptal wall but the pressure inside the pseudoaneurysm sac was not high enough for bubbles to enter the RV. Anteroseptal wall was intact as seen on subcostal echo images but pseudoaneurysm sac was still visible in the RV illustrating the large sized pseudoaneurysm and its relationship with the RV (Figure 5). Short axis views showed that the basal inferior and inferoseptal wall had a hole in it and was bulging out as a pseudo aneurysm into the adjacent RV and the inferior wall of the RV adjacent to the septum was also suspicious for pseudoaneurysm as later confirmed surgically (Figure 6). 
A CT angiogram confirmed that the LV had thinning of the inferior wall and a very large pseudoaneurysm was projecting into the pericardium and also towards the RV with the adjacent inferior wall of RV bulging inferiorly as well (Figure 7). The opening of LV pseudoaneurysm through a hole into the RV pseudoaneurysm creating a VSD could also be seen on CTA (Figure 8). The open surgical repair confirmed the above remarkable scenario with both LV and RV involved with pseudoaneurysms and the LV pseudoaneurysm sac communicated with RV creating an unusual VSD.

As above, the patient was taken to the operating room and the open surgical evaluation revealed a very large and complex left ventricular pseudoaneurysms and even an adjacent RV pseudoaneurysm. OR findings confirmed the echo and CT findings that both the inferior/inferoseptal walls of the left ventricle had blown out into a large pseudoaneurysm. The adjacent inferior right ventricle was involved by the pseudoaneurysm and the 2 ventricles communicated through the pseudoaneurysm sac.

Our patient's echocardiogram pre surgery with massive hole in inferoseptum (Figure 9) can be contrasted with his echocardiogram immediately post surgery with intact inferoseptum (Figure 10). On post surgical echo imaging, he had no pseudoaneurysm and no shunting. Subsequent to the surgery, our patient underwent rehabilitation post discharge. He has been seen in our outpatient cardiology office more than two years after surgery and he has been asymptomatic with excellent exercise tolerance.

Discussion:

We present an unusual case of a huge pseudoaneurysm involving both the basal inferior wall and inferior septum but even the adjacent RV inferior wall. The pseudoaneurysm ruptured straight into the right ventricle instead of the pericardial cavity and this caused a tiny VSD instead of tamponade (9).

Mechanical complications of FWR, VSD and papillary muscle rupture have become rarer in the reperfusion era but still present with devastating complications of MI like cardiogenic shock (1,2). Patients often present with single-vessel coronary artery disease and usually do not have a positive history for a previous myocardial infarction $(1,2,3)$. The incidence of post infarction angina in these patients is significantly greater than in patients without ventricular rupture $(1,2,3)$. Delay in treatment and continued physical activity post infarction increases the risk of ventricular rupture $(1,2,3)$.

Imaging is critical to diagnosis of mechanical complications of acute MI. Our echocardiographic images after the patient's presentation showed that the LV inferoseptal wall with an unusual pseudoaneurysm that bulged into the adjacent RV. The color Doppler suggested shunting through the LV pseudoaneurysm into the RV creating an unusual VSD. The CT angiograms corroborated the echo findings. Subsequently, our patient went for surgical repair of pseudoaneurysm and the open surgical findings confirmed the imaging findings that there was a massive LV pseudoaneurysm from the inferior and inferoseptal walls, the RV was involved with pseudoaneurysm of its inferior wall adjacent to the septum and a communication with LV pseudoaneuysm sac and the RV causing a previously described VSD was seen.

Conclusion:

The co-occurrence of VSD with pseudoaneurysm formation or concurrent LV and RV double rupture are very rare but have been reported $(11,12)$. The clinical scenario in our patient of having concomitant pseudoaneurysm of LV and adjacent RV leading to an unusual VSD with communication between the two ventricles created by a tear in pseudoaneurysm sac has never been reported in the past. Our patient's unique pathology was suggested by echocardiography and CT angiographic findings and then confirmed during the open surgical repair.

References:

1. Lopez-Sendon J, Gurfinkel EP, Lopez de Sa E, Agnelli G, Gore JM, Steg PG, Eagle KA, Cantador JR, Fitzgerald G, Granger CB. Factors related to heart rupture in acute coronary syndromes in the Global Registry of Acute Coronary Events. Eur Heart J 2010. 31:1449-1456. 
2. Koeda $\mathrm{Y}$, Itoh $\mathrm{T}$, Ishikawa $\mathrm{Y}$, et al. A multicenter study on the clinical characteristics and risk factors of in-hospital mortality in patients with mechanical complications following acute myocardial infarction [published online ahead of print, 2020 Apr 1]. Heart Vessels . 2020;10.1007/s00380-02001586-0. doi:10.1007/s00380-020-01586-0

3. Davis N, Sistino JJ. Review of ventricular rupture: key concepts and diagnostic tools for success. Perfusion $2002 ; 17: 63-7$.

4. Craig Frances, Anthony Romero, Deborah Grady. Left ventricular pseudoaneurysm. Journal of the American College of Cardiology Sep 1998, 32 (3) 557-561; DOI:10.1016/S0735-1097(98)00290-3.

5. Mark C.K. Hamilton, Jonathan C.L. Rodrigues, Robin P. Martin, Nathan E.Manghat, Mark S. Turner. The In Vivo Morphology of Post-Infarct Ventricular Septal Defect and the Implications for Closure. JACC: Cardiovascular Interventions Jun 2017, 10 (12) 1233-1243; DOI:10.1016/j.jcin.2017.03.042.

6. Bates ER. Reperfusion therapy reduces the risk of myocardial rupture complicating ST-elevation myocardial infarction. J Am Heart Assoc. 2014;3(5):e001368.

7. Vlodaver Z, Coe 11. Edwards IE. True and false left ventricular aneurysms. Propensity for the latter to rupture. Circulation 1975;51:567-72.

8. Catherwood E, Mintz GS, Kotler MN, Parry WR, Segal BL. Two-dimensional echocardiographic recognition of left ventricular pseudoaneurysm. Circulation 1980;62:294-303.

9. Gatewood RP, Nanda NC. Differentiation of left ventricular pseudoaneurysm from true aneurysm with two dimensional echocardiography. Am J Cardiol 1980;46:869-78.

10. Recusani F, Raisaro A, Sgalambro A, et al. Ventricular septal rupture after myocardial infarction: diagnosis by two-dimensional and pulsed Doppler echocardiography. Am J Cardiol 1984;54:277-81.

11. Hamilton K. Ellenbogen K. Lowe JE. Kisslo J. Ultrasound diagnosis of pseudoaneurysm and contiguous ventricular septal defect complicating inferior myocardial infarction. J Am Coil Cardiol 1985;6:11 60-3.

12. Chen S-J, Zhang C, Meng Q-T, Peng Y, Chen M. Sudden death due to ventricular double rupture as a complication of inferior acute myocardial infarction: A case report. Bil. J, ed. Medicine . 2016;95(52):e5757. doi:10.1097/MD.0000000000005757.

Figures and Videos (videos in Supplementary Material) :

Figure 1 and Video 1. 4 chamber view showing the basal inferior septum (INFEROSEP) with a large pseudoaneurysm (PSA) creating a VSD and bulging into the right ventricle.

Figure 2 and Video 2: Subcostal view again showing the large pseudoaneurysm coming off the base of the inferior septal wall.

Figure 3 and Video 3: Subcostal view showing that through the pseudoaneurysm sac, there is a VSD leading to a communication between the $\mathrm{LV}$ and the RV

Figure 4 and Video 4. Four chamber view again supporting the unusual VSD through the sac of the pseudoaneurysm into the RV as well as associated tricuspid regurgitation.

Figure 5 and Video 5: Dramatic subcostal view of the pseudoaneurysm showing that the anterior septum (ANTSEP) is intact and the bulging pseudoaneurysm from the inferoseptal wall is seen in the right ventricle.

Figure 6: Short axis view showing that the basal inferior and inferoseptal wall has a hole in it and is bulging out as a pseudo aneurysm into the adjacent RV

Figure 7. CT angiogram (CTA) showing that the basal LV inferior wall is thinned out with an inferior wall pseudoaneurysm and adjacent RV inferior wall aneurysm

Figure 8. CT angiogram showing the pseudoaneurysm part of the LV that communicates via a hole (VSD) into the RV pseudoaneurysm

Figure 9. Presurgical echo subcostal images illustrate the narrow neck in inferoseptal wall into a giant pseudoaneurysm

Figure 10. Post surgical repair now showing an intact inferoseptal wall. 

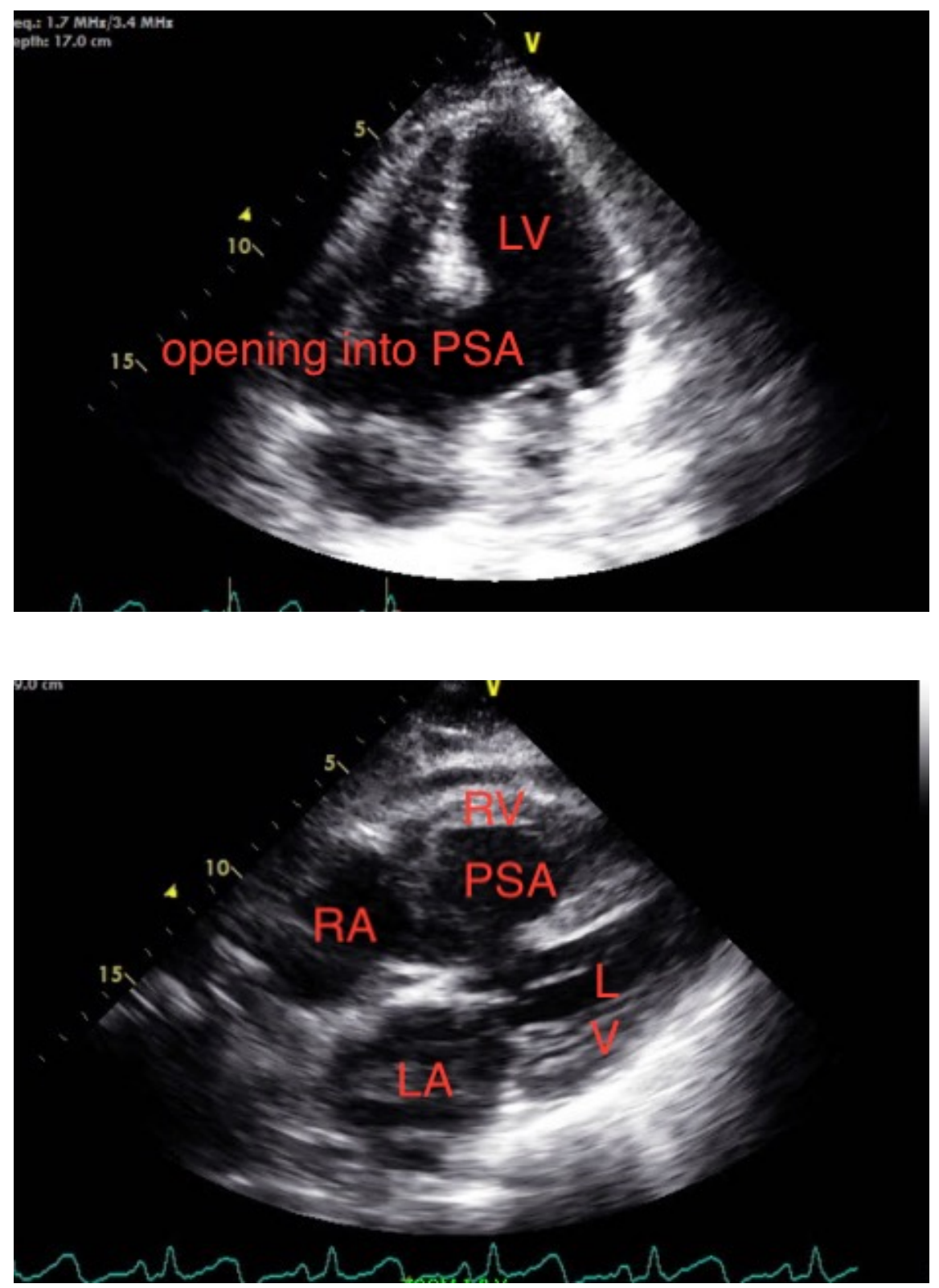

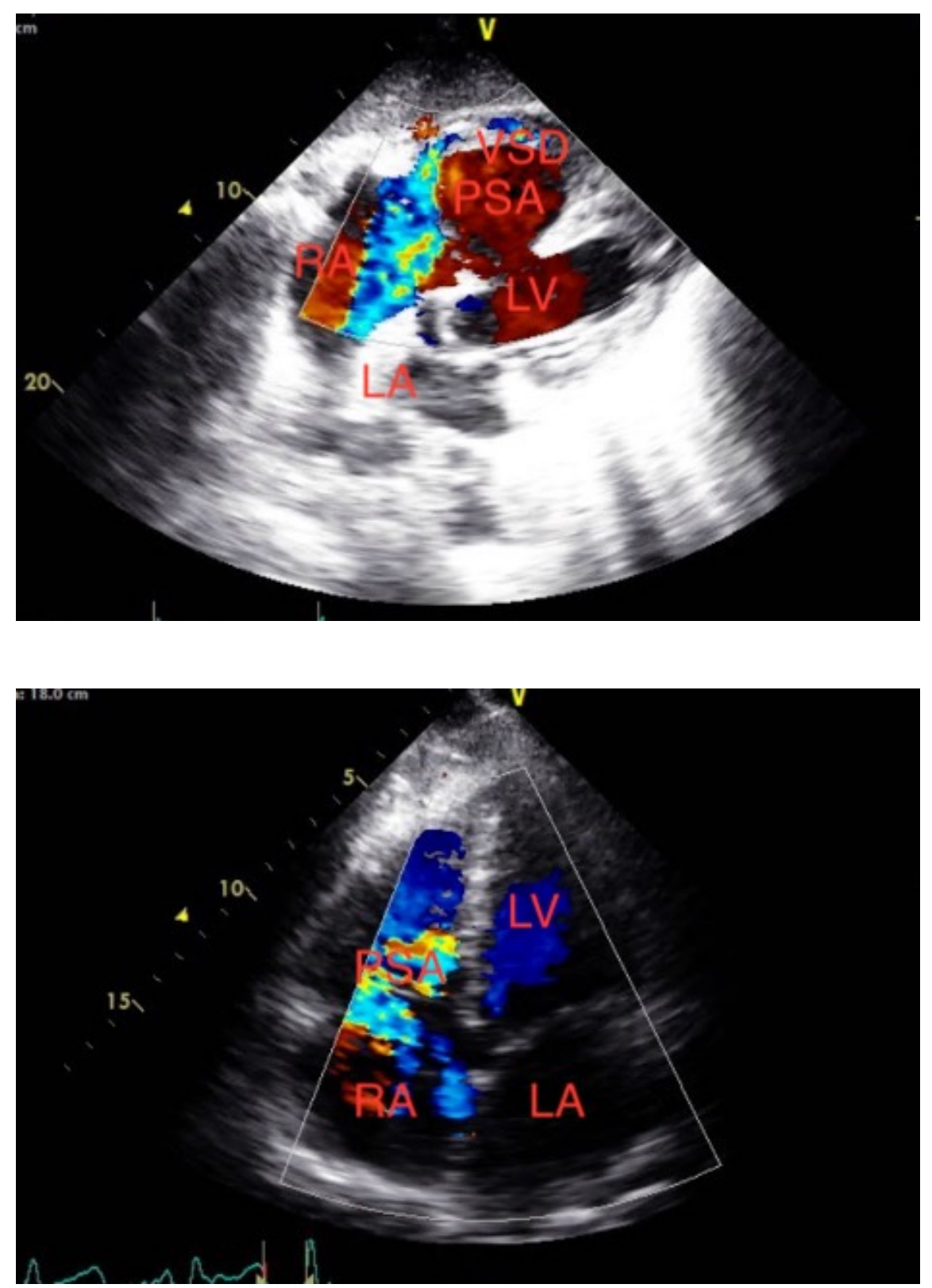

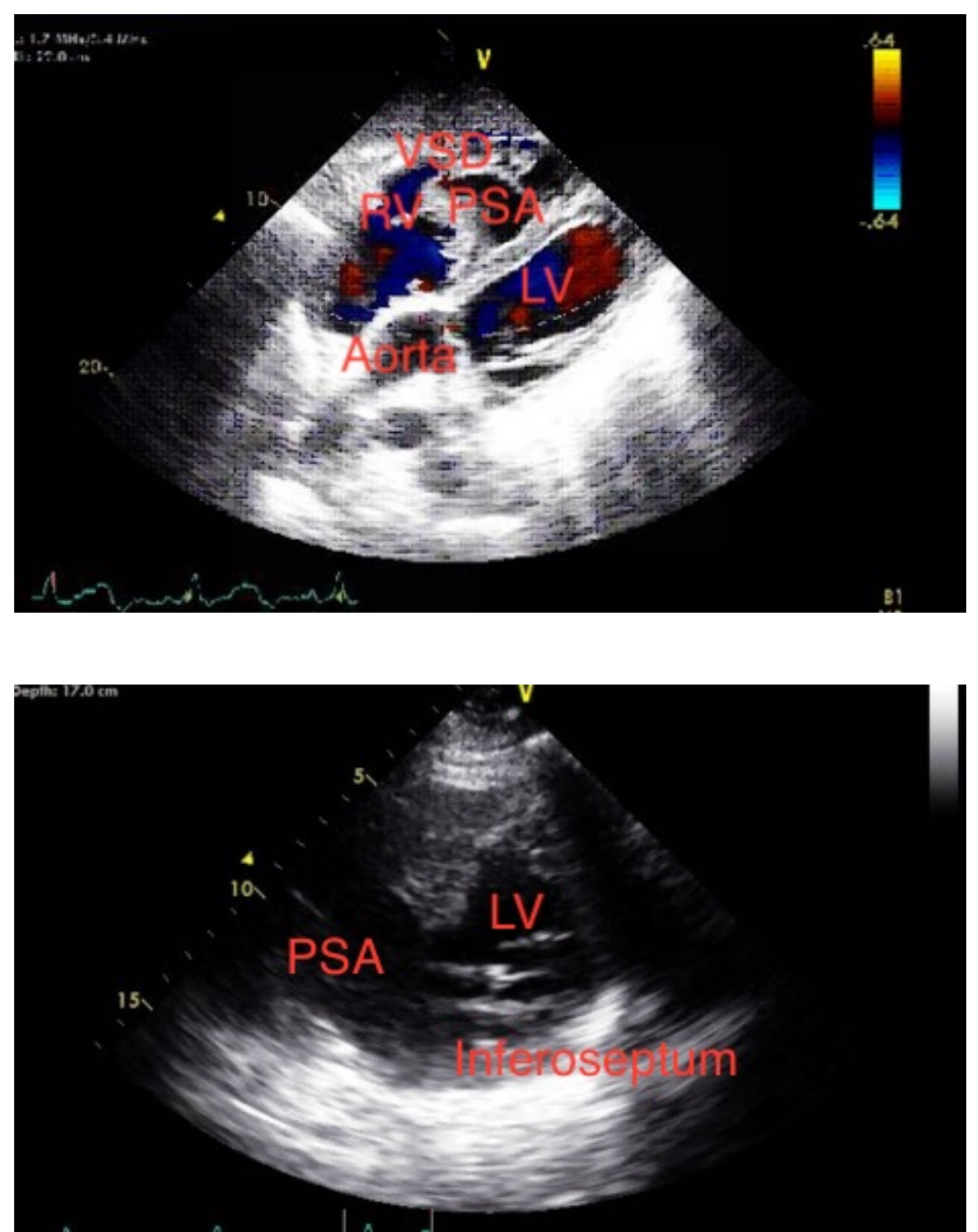

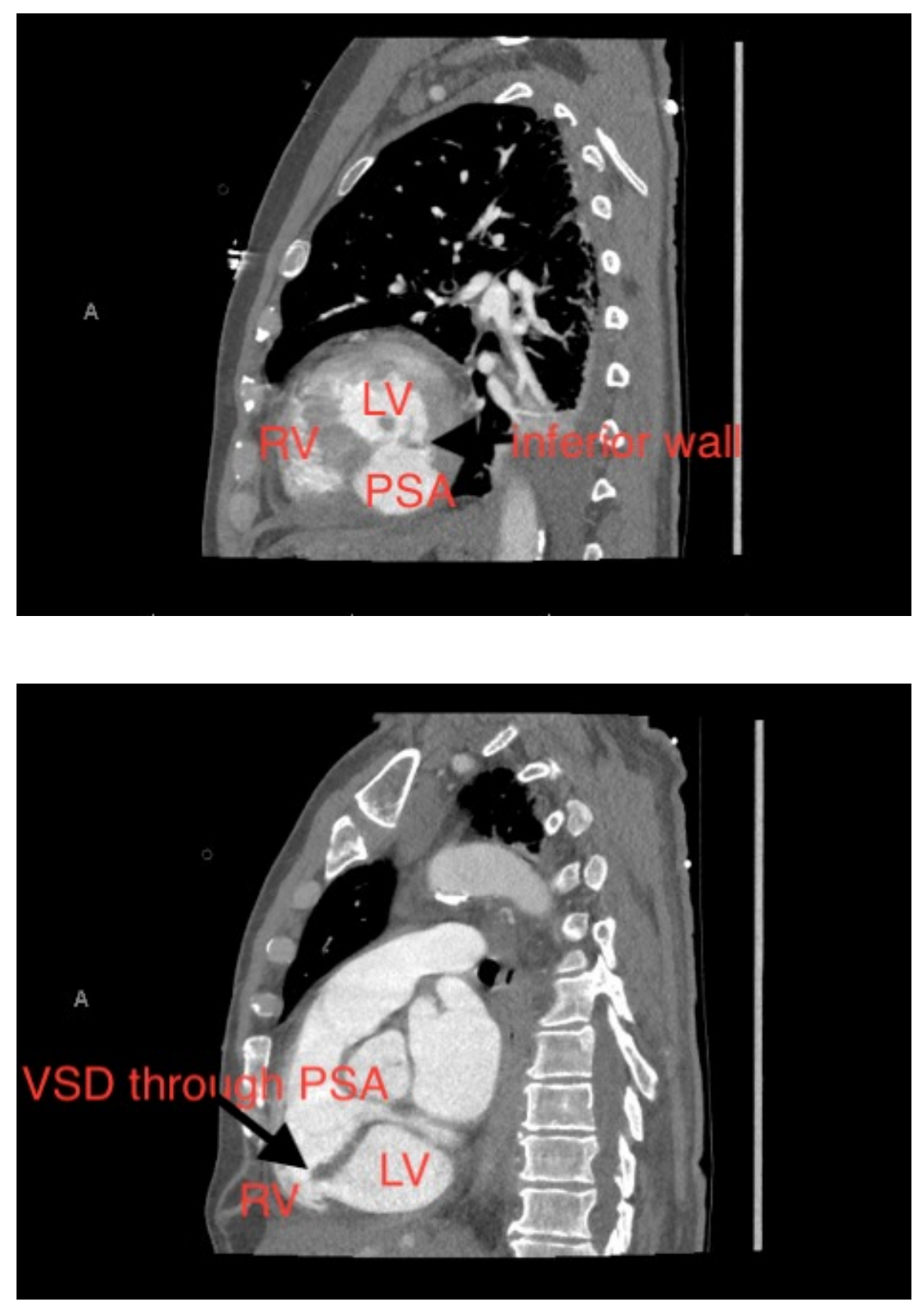

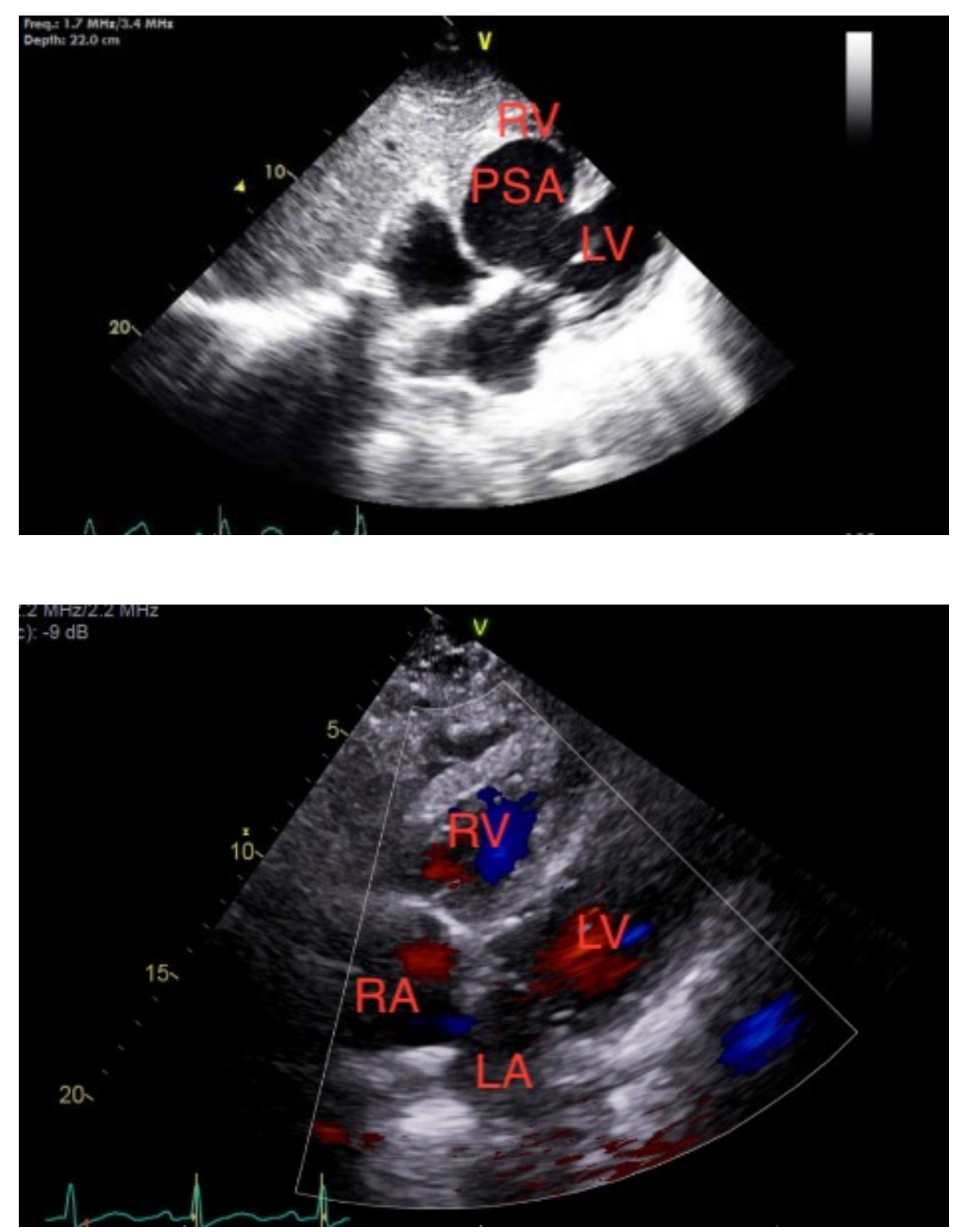